\title{
Images of the Seabed of the Gulf of Gdańsk Obtained by Means of the Parametric Sonar
}

\author{
E. KOZACZKA ${ }^{a}$, G. GRElowskA ${ }^{a}$ AND S. KOZACZKA ${ }^{b}$ \\ ${ }^{a}$ Polish Navy Academy Gdynia \\ ${ }^{b}$ Gdańsk University of Technology, Narutowicza 11/12, 80-223 Gdańsk, Poland
}

\begin{abstract}
The main goal of this paper is to present results of the experimental investigation of the seabed by means of parametric echosounder in the form of chosen images. The phenomena of nonlinear interaction between two parallel beams of high intensity gives as a results very narrow beam of low frequency wave, that enables to penetrate the sea bottom. The first step of our investigations was calibration of all the elements of the measuring system. In the second step the marine trials were carried out. For this purpose the mobile measuring set up was installed on the board of sailing boat. The details about the measuring devices can be found below. The main goal of these investigations was to determine the structure of the seabed for different places of the Gulf of Gdańsk. The special features of parametric generation of sound beam allow to penetrate the bottom for more then several meters. At the end of the paper, a set of images that illustrated the results of investigations has been presented.
\end{abstract}

PACS numbers: 43.30.Ma, 43.50.Pc, 43.25.Jh

\section{Introduction}

The study of the seabed by means of acoustic methods has been carried out for decades. Most of the obtained results cover the topic of the geometry of the seabed. At present, mapping the surface of the sea is a relatively easy, especially while using the multibeam sonars [1-3].

Sonars used for the observation of the seabed, including the side and multibeam sonars, enable receiving the information about the shape of the seabed and objects lying on it [4]. Despite their impressive resolution, it is impossible to obtain information neither about the stratification of the sediments nor about the objects buried in the seabed. Since the attenuation of the elastic wave is much higher in sediments than in the water, the waves that are of relatively high frequency are not able to penetrate the bottom sediments. Waves of relatively low frequencies can propagate deeper into them. A device producing waves on the basis of the parametric effect enables the enlargement the possibility of studies of the seabed stratification and searching for objects buried in the seabed $[5,6]$. In general, the phenomenon can be described by two waves, which are propagated in the same area and their wave vectors are parallel to each other. The frequency of these waves is high enough to produce so called secondary waves, including the wave of the frequency equal to the difference of frequencies of primary waves, called sometimes the difference frequency wave. Generated wave has the characteristics of primary waves including width of the beam pattern and depth resolution, which determined the spatial resolution.

\section{The simplified theoretical description} of the phenomena of the parametric generation of the elastic waves in water

The principle of parametric wave generation is a nonlinear interaction of two collinear waves of high intensity propagated in the same direction. Before describing the generation of the waves including the generation of the low frequency wave, the substance of nonlinear propagation of the wave will be presented by showing the example of harmonic wave of high frequency. Nonlinear properties of the environment, especially the ones described by the equation of the state of the medium, that connect the pressure and the density of the medium cause that the propagated wave of the finite amplitude is distorted. This distortion increases to a certain point causing that apart from the original wave, called primary one, appear secondary waves of frequencies that are multiple of the original frequency of the primary wave. The illustration of this phenomena are the shapes of the waves at points lying in the beam axis and corresponding to the different distance from the source (Fig. 1).

Theory of parametric wave generation is very complex. It leads to the equation derivated from the general law of mass conservation, law of conservation of momentum in the form of the Navier-Stoke's equation, the equation of state and the equation of energy. After a number of simplifications the relatively simple equation called the $\mathrm{KZK}^{*}$ is received. This is still a complex equation, however, relatively friendly for receiving some numerical solutions for the certain boundary conditions.

In this paper, to illustrate the parametric effect the simplified form of KZK equation, called Burger's equation, was used:

$$
\frac{\partial p^{\prime}}{\partial x}-\frac{\varepsilon}{\rho_{0} c_{0}^{3}} p^{\prime} \frac{\partial p^{\prime}}{\partial \tau}-\frac{b}{2 \rho_{0} c_{0}^{3}} \frac{\partial^{2} p^{\prime}}{\partial \tau^{2}}=0
$$

where: $x$ - the axis perpendicular to the plane of the radiation, $p^{\prime}(\tau)$ - the dynamic component of the pressure

\footnotetext{
* (KZK - from the surnames of Khokhlov, Zabolotskaya and Kuzniecow)
} 

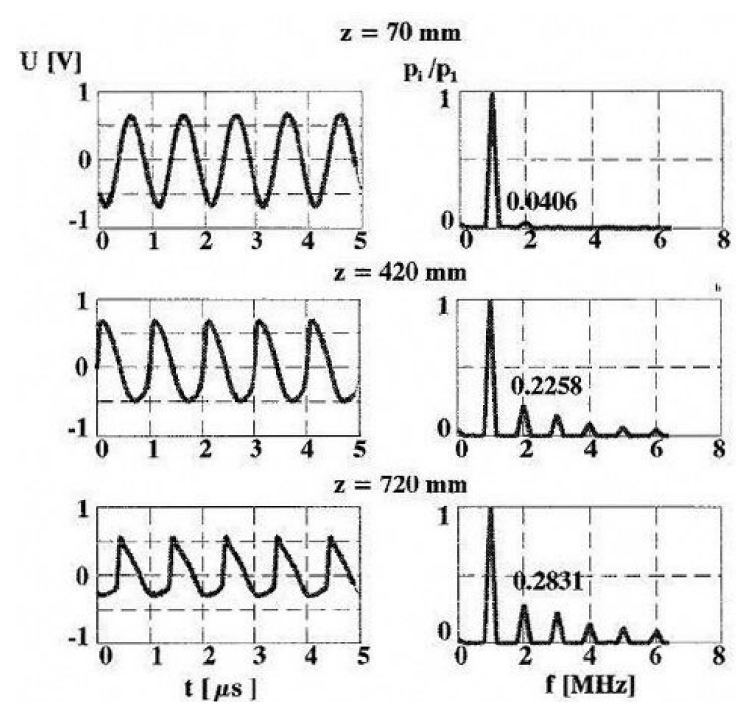

Fig. 1. The shape and the spectrum of the wave in the axis of the beam set for different distances from the source.

of the elastic wave, $p_{0}$ - static pressure in the medium, $\tau=t-\frac{x}{c}$ - time retarded, $\varepsilon-$ the coefficient of nonlinearity of the medium, $\rho_{0} c_{0}$ - the density and wave speed in the equilibrium medium, $b$ - the coefficient of linear dissipation of the wave energy.

The solution of Eq. (1) is sought by the method of successive approximations

$$
p^{\prime}=p_{1}+p_{2} \text {. }
$$

The solution could be obtained for $R_{e a}<1$ (where $R_{e a}=\frac{p}{b \omega}, \omega$ - the angular frequency of wave) which indicates the little influence of nonlinear effects. The value $R_{e a} \gg 1$ is characteristic for the high wave distortion.

If it has been assumed that the boundary condition will be in the following form for $x=0$ :

$$
p_{1}\left(x_{1} \tau\right)=p_{01} \sin \omega_{1} \tau+p_{02} \sin \omega_{2} \tau,
$$

where: $p_{01}$ - the amplitude of the wave of the angular frequency $\omega_{1}, p_{02}$ - the amplitude of the wave of the angular frequency $\omega_{2}$, the part of the solution received for the Eq. (1) under the conditions (3) and the assumption (2) describing only the difference frequency wave is as follows:

$$
\begin{aligned}
& p_{2}(x \tau)=\frac{\varepsilon p_{01} p_{02}\left(\omega_{1}-\omega_{2}\right)}{2 b \omega_{1} \omega_{2}} \\
& \quad \times\left[\exp \left(\frac{b\left(\omega_{1}^{2}+\omega_{2}^{2}\right)}{2 \rho_{0} c^{3}} x\right)-\exp \left(\frac{b\left(\omega_{1}-\omega_{2}\right)^{2}}{2 \rho_{0} c_{0}^{3}} x\right)\right]
\end{aligned}
$$

for $\omega_{1}>\omega_{2}$.

On the basis of the equation above the pressure of the wave of frequency equal to the difference between frequencies of primary waves can be estimated. The beam of secondary wave combines advantages of primary waves, narrow beam pattern and advantages of the low frequency wave, relatively low sound energy attenuation in the sediments. Those features allow to obtain sound beam of a good spatial resolution that is able to propagate into sediments. Basing on nonlinear acoustics theory, describing the generation of the difference frequency wave, parametric sonars and echosounders are designed.

\section{The experimental investigation in the Gulf of Gdańsk}

The bottom structure was investigated using the parametric echosounder SES-2000 produced by Innomar. It is a non linear transducer source which simultaneously transmits two signals of slightly different high frequencies at high sound pressures. Nonlinear interactions generate new frequencies in the water, one of them being the difference frequency that has a bandwidth similar to the primary frequency. Both the primary high frequency (HF) signal $(100 \mathrm{kHz})$ and the secondary low frequency (LF) signal (6 to $15 \mathrm{kHz}$ ) are recorded. Penetration can reach up to a few tens of meters in soft sediments. Advantages of the parametric acoustic system include (1) small beam width at low frequencies, (2) deep penetration with high resolution of sediment layers and objects, and (3) accurate depth measurements with the high frequency signal.

The parametric echosounder (Fig. 2) is the main part of the measuring system additionally equipped in several indispensable devices to ensure the great quality of measurements, as well as high accuracy in positioning of the vessel. It is used in combination with a motion sensor (MRU-Z, Kongsberg) to correct for swell movement. Data processing is carried out with the processing software "ISE 2.9" which allows layer editing and export to ASCII data, extended signal processing, data conversion and data export, tide, water sound velocity, and GPS z-level correction.

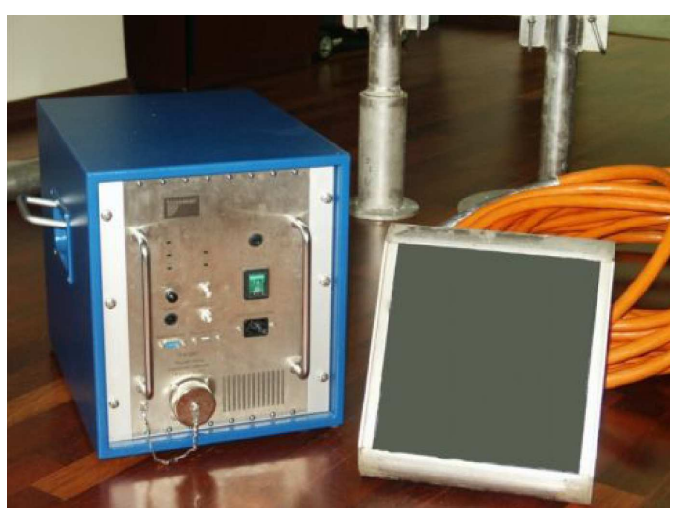

Fig. 2. SES-2000 compact echosounder.

Measurements are done from the boat that is navigated using the differential Global Positioning System (DGPS). The average navigational accuracy is estimated to be better than $1.5 \mathrm{~m}$. Moreover, the position was controlled using radar Nobeltec operating with Visual Navigation Suite Admiral MAX. The set-up used in investigation is shown in Fig. 3. 


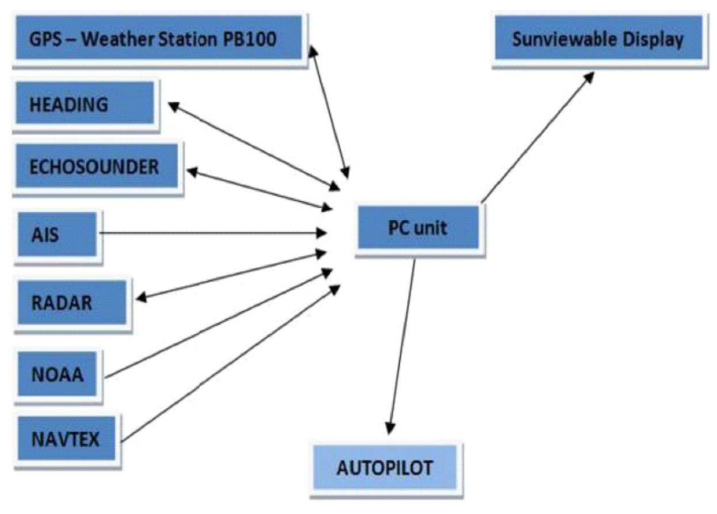

Fig. 3. Measurement setup used during sea bottom structure investigation.

Additional equipment, shown in Fig. 3, such as the Automatic Identification System AIS, the NOAA (National Oceanic and Atmospheric Administration) weather station, or Navtex receiver, serves to ensure safety during research at sea, monitoring navigational and meteorological warnings.

Sea bottom investigation in the Gulf of Gdańsk is carried out using two complementary devices parametric echosounder and multibeam echosounder. Both of them are equipped with GPS and Heading as positioning system, and with the MRU-Z system compensating the impact of the vessel's movement on results of measurements (Figs. 4, 5).

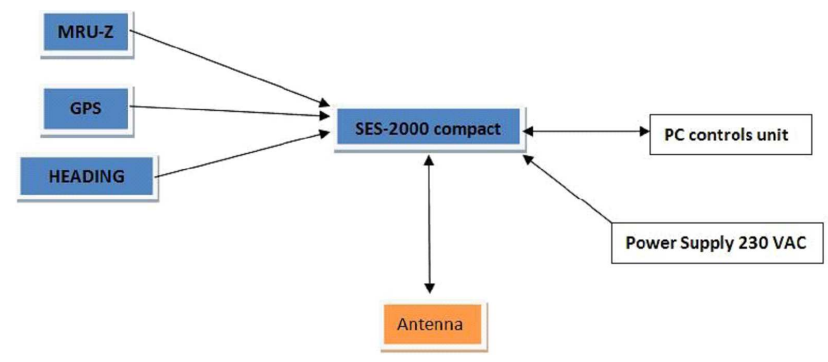

Fig. 4. Configuration of parametric echosounder SES-2000 compact.

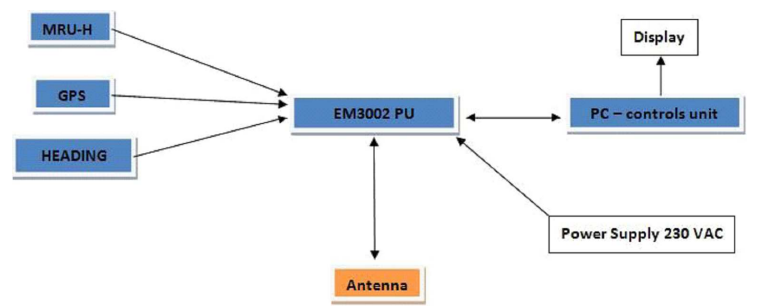

Fig. 5. Configuration of EM-3002 multibeam echosounder.
During investigations of the bottom structure of the Gulf of Gdańsk, the marine trials were carried out. Their range of research is shown on the map in Fig. 6.

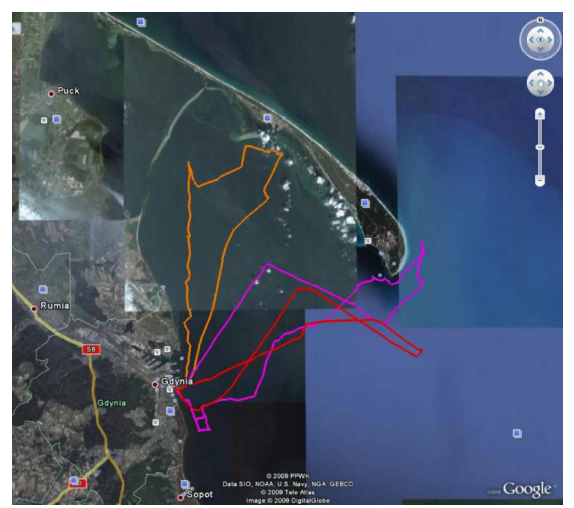

Fig. 6. Map with selected measurement tracks.

The main goal of these investigations was to determine the structure of the seabed for different places of Gdańsk Bay.

The diversity of seabed characterization techniques caused problems with using them in a complementary manner. Each technique provides an image of the seabottom that is filtered by the technique itself. In Fig. 7, is shown an underwater image of a wreck, obtained by means of multi beam echosounding technique. The shape of the wreck is well visible against the background of the seabed.

A typical image of wreck obtained using parametric echosounder is shown in Fig. 8. Looking at both of those images, thesis that each technique provides another information, which is specific for itself can be confirmed.

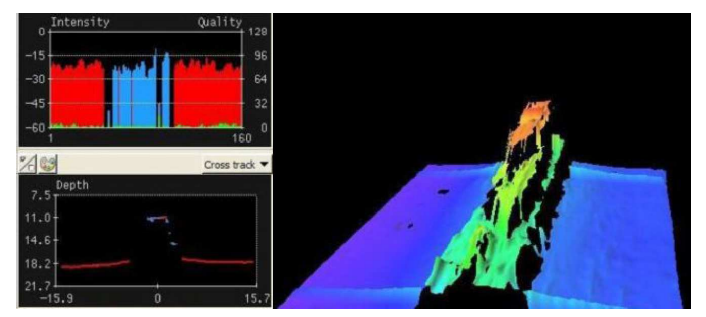

Fig. 7. Example of wreck sounding with EM-3002.

The special features of parametric generation of sound beam allow to penetrate the bottom for much more then several meters. Data collected during observation allow to assess the penetration properties of the equipment.

High-resolution sediment-echosounding allows distinguishing between sediment layers of different impedance. In the Gulf of Gdańsk most common is an echogram characterized by numerous distinct, closely spaced and continuous parallel horizontal reflectors. There are particularly strong major reflectors within the vertical sequence which can be traced over several kilometers distance (Fig. 9). Acoustic penetration was at given condi- 


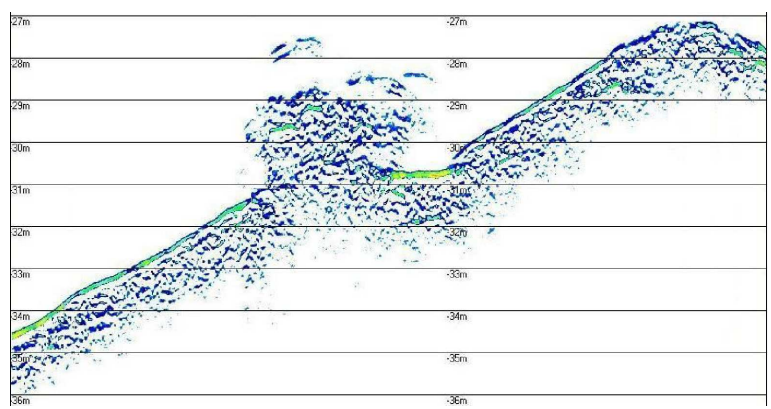

Fig. 8. Echogram of a wreck from parametric echosounder SES-2000 compact.

tions of about 30 meters. The fine structure of deeper layer of sediments is reflected in the image.

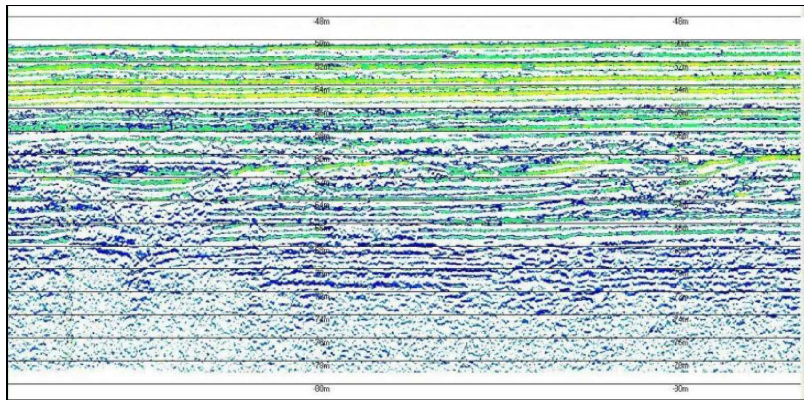

Fig. 9. Seabed structure typical for many areas in the Gulf of Gdańsk region.

The depth of penetration depends on geoacoustic parameters of bottom sediments. For sediments of great acoustic impedance and at the same time great attenuation parametric beam reach only few meters depth below water-sediments border, as it is shown in Fig. 10.

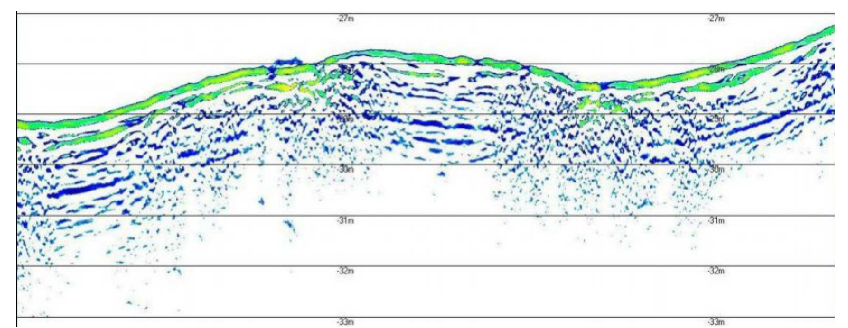

Fig. 10. Structure of upper layer sediments of bottom composed of well reflected material.

The parametric echosounder allows to carry out geological research of the upper layer of the seabed remotely.
An interesting example of rapid change in the seabed structure is observed in the part of the Gulf of Gdańsk known the Puck Bay (Fig. 11). This way, spatial distribution of the seabed sediments could be established more precisely than by any other method of seabed investigation.

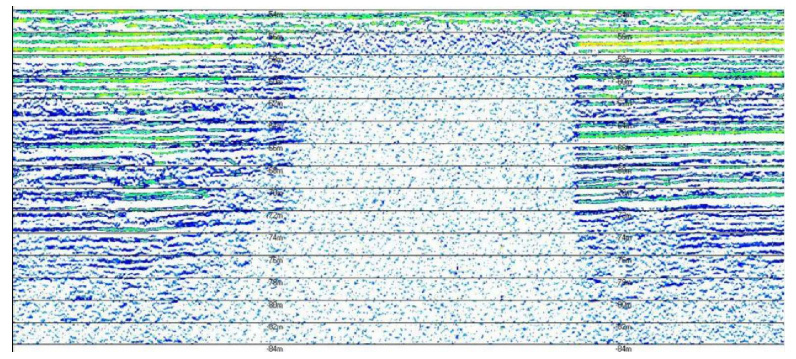

Fig. 11. Change in seabed structure in the Puck Bay area.

\section{Conclusions}

Taking into account the experimental results, following conclusions can be drawn. The parametric echosounder is a good tool for examination of the acoustical feature of the sea bottom up to $10 \mathrm{~m}$. The depth of penetration depends on the geoacoustic parameters of the seabed sediments. The acoustic beam penetrates the seabed with the resolution good enough to evaluate the layered structure of the sea bottom and to look for objects buried in the sediments.

\section{Acknowledgments}

The investigation was supported by the Ministry of Science and Higher Education (Grant No R00 012 01).

\section{References}

[1] D.R. Jackson, M.D. Richardson, High-Frequency Seafloor Acoustics, Springer, (2007).

[2] I. Karasalo, P. Skogqvist, in: Acoustic sensing techniques for the shallow water environment, Springer, (2006).

[3] P. Moren, et al., in: Buried waste in the seabed, Springer, (2007).

[4] M. Zakharia, J. Dybedal, in: Buried waste in the seabed, Springer, (2007).

[5] G. Grelowska, E. Kozaczka, Hydroacoustics 11, 105 (2008).

[6] E. Kozaczka, G. Grelowska, S. Kozaczka, Sounding of the seabed of the Gulf of Gdansk by the means of the parametric sonar, Proceedings of 16 ICSV Cracow, 2009. 